\title{
Synthesis of Polythioesters and Polythiohydrazides
}

\author{
Yasushi Kishimoto, Kohei Sanui, and Naoya Ogata \\ Department of Chemistry, Sophia University, \\ Chiyoda-ku, Tokyo, Japan.
}

(Received January 26, 1972)

\begin{abstract}
Various polythioesters and polythiohydrazides were synthesized by the polycondensation of dithiocarboxylic acid- $O, O$-diethyl esters with dihydrazides and diols in solution at a relatively low temperature. The polycondensation reaction took place with a much slower rate than that with diamines and the reactivity of dithiocarboxylic acid- $O, O$-diethyl ester with nucleophiles was in the following order: diamine $>$ dihydrazide $>$ diol.
\end{abstract}

KEY WORDS Polycondensation./ Polythiohydrazide / Dihydrazide /

Diol /

It has been previously reported ${ }^{1}$ that the polycondensation reactions of dithiocarboxylic acid$O, O$-diethyl ester with diamines take place at room temperature to form high-molecular-weight polythioamides in solution. In these reactions, it has been found that the rates of polycondensation are influenced by the solvents, presumably due to the changes in nucleophilicity of the amino group in solution, and that the structures of resulting polythioamides are also dependent on the solvents, for instance dimethyl sulfoxide is an effective solvent to yield a linear polymer, while an in- soluble polymer with a cross-linked structure is obtained in ethanol.

Thus, other weak nucleophiles such as dihydrazide or diols may react with dithiocarboxylic acid- $O, O$-diethyl ester at a relatively low temperature to form corresponding polymers when a suitable solvent is selected for the polycondensation.

This paper deals with the polycondensation of dithiocarboxylic acid-O,O-diethyl ester with various diols or dihydrazides as shown below.

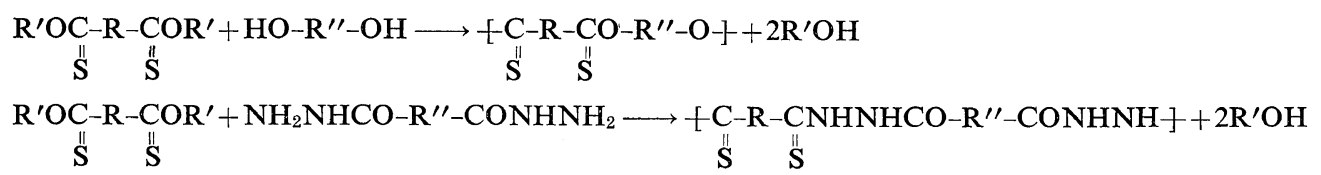

EXPERIMENTAL

\section{Materials}

Commercially available ethylene glycol, 1,4butanediol, and bisphenol A of first grade reagent quality were further purified by distillation under reduced pressure prior to use. Solvents such as dimethylformamide(DMF), dimethylacetamide (DMAc), $N$-methyl-2-pyrrolidone(NMP), hexa-
methylphosphoramide(HMPA) were purified by distillation.

\section{Synthesis of Monomers}

Dihydrazides were synthesized by the reaction of diesters with hydrazine in methanol, followed by recrystallization from methanol-water.

Dithio-isophthalic acid-O,O-diethyl ester(DTIP) was synthesized from dinitrile according to the method previously reported. ${ }^{1}$

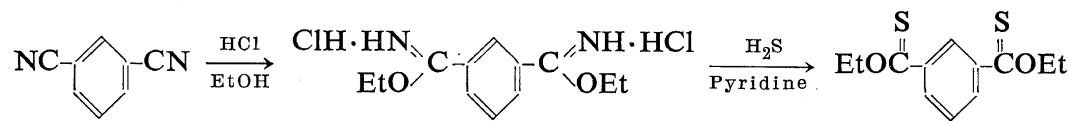

$$
\begin{aligned}
& \text { yield } 71 \% \text {, mp } 295-298^{\circ} \mathrm{C} \text {. }
\end{aligned}
$$




\section{Solution Polycondensation}

Equal molar quantities of DTIP and diols or dihydrazides were dissolved in various solvents in the presence of $5 \mathrm{~mol} \%$ of lithium ethoxide and the solutions were kept at definite temperatures. The rate of the polycondensation was determined by measuring the amount of ethanol eliminated in a given period from the solution by means of gas-chromatography or by titration of the residual hydrazide with $0.1-N \mathrm{HCl}$ in solution.

After the polycondensation was completed, the solution was poured into water and resulting polymers were filtered, washed with benzene, and dried.

\section{Characterization}

The structures of the resulting polythioesters or polythiohydrazides were identified by elementary analysis and infrared spectrum. Solution viscosities of the polymers were measured in DMSO at a concentration of $0.5 \mathrm{~g} / \mathrm{d} l$ at $30^{\circ} \mathrm{C}$. Melting points of the polymers were measured by differential thermal analysis.

Elementary analyses of a polymers obtained were in close agreement with the expected structure as follows.

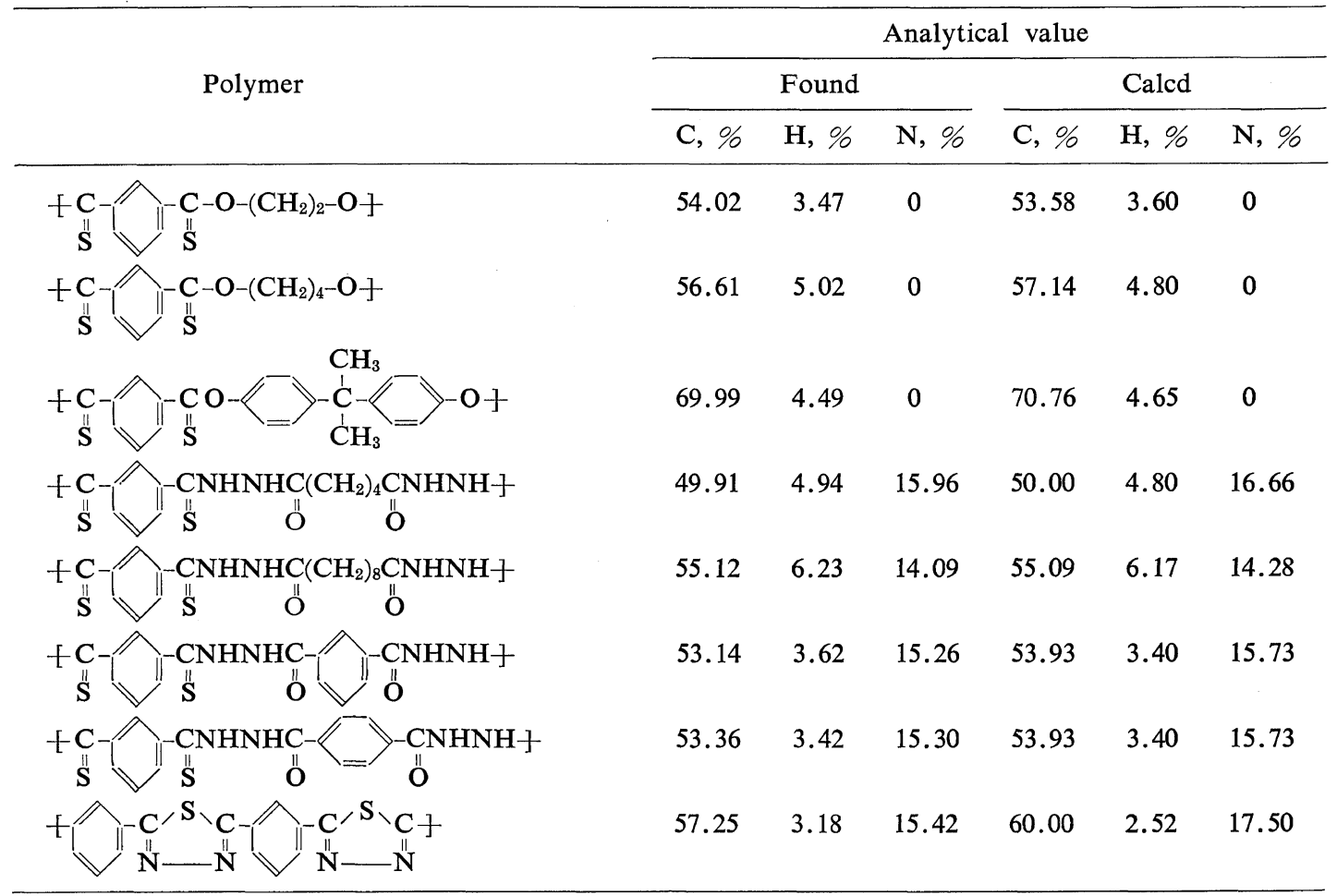

\section{RESULTS AND DISCUSSION}

\section{Polythioesters}

The rates of polycondensation of DTIP with 1,4-butanediol or bisphenol $\mathrm{A}$ in various solvents are shown in Figures 1 and 2, respectively, from which it is seen that DMSO is an effective solent to facilitate the polycondensation although the rates are much slower than the rate ${ }^{1}$ with aliphatic diamines which is completed within $5 \mathrm{hr}$. All the polycondensation reactions did not proceed to completion. However, no equilibrium between monomer and polymer was reached because the polycondensation reaction took place very slowly even after one month.

Results of the polycondensation of DTIP with various diols are summarized in Table I. Yields and solution viscosities of the resulting polythioesters were not satisfactory in comparison with the results of polythioamide from DTIP and 


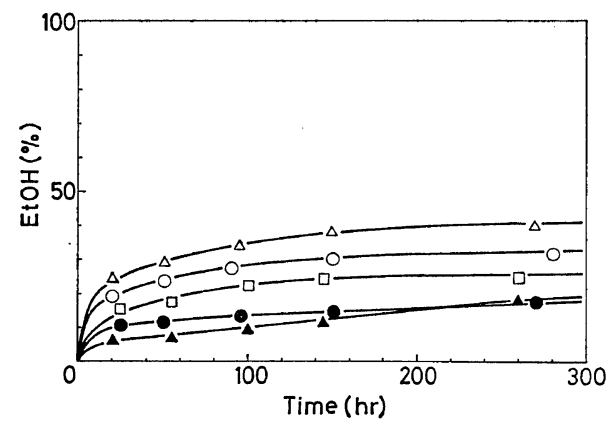

Figure 1. Rates of polycondensation of DTIP with 1,4-butanediol in various solvents at $60^{\circ} \mathrm{C}: \bigcirc$, DMF; $\triangle$, DMSO; $\square$, DMAc; ๑, NMP; $\triangle$, HMPA; monomer concn, $0.25 \mathrm{ml} / l$; catalyst, EtOLi, $5 \mathrm{~mol} \%$.

diamines. The structure of polythioesters was identified by elementary analysis and infrared spectra as shown in Figure 3.

All of the polythioesters were soluble in DMSO and DMF and no insoluble polymers with crosslinked structure were obtained. Melting points of the resulting polythioesters were lower than those of the corresponding polyesters.

No polythioesters were obtained by the inter-

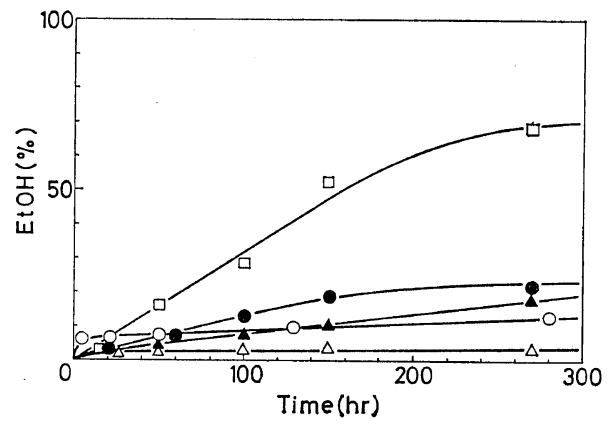

Figure 2. Rates of polycondensation of DTIP with bisphenol $\mathrm{A}$ in various solvents at $60^{\circ} \mathrm{C}$ : $\bigcirc$, DMF; $\triangle$, DMAc; $\square$, DMSO; •, NMP; $\Delta$, HMPA; monomer concn, $0.25 \mathrm{~mol} / l$; catalyst, EtOLi, $5 \mathrm{~mol} \%$.

facial polycondensation of DTIP with 1,4-butanediol or bisphenol A under the following reaction condition: DTIP concentration $0.5 \mathrm{~mol} / l$ in chloroform, diols in water, $30^{\circ} \mathrm{C}, 50 \mathrm{hr}$.

\section{Dithiohydrazides}

Rates of the polycondensation of DTIP with isophthalic acid dihydrazide were determined in various solvents as shown in Figure 4. It was found that DMSO was an effective solvents to

Table I. Polycondensation of DTIP with diols under various conditions ${ }^{a}$

\begin{tabular}{|c|c|c|c|c|c|c|c|c|}
\hline $\begin{array}{c}\text { Diol } \\
\text { HO-R-OH }\end{array}$ & Solvent & $\begin{array}{c}\text { Concn, } \\
\text { mol } / l\end{array}$ & $\underset{{ }^{\circ} \mathrm{C}}{\mathrm{Temp}}$ & $\begin{array}{c}\text { Time } \\
\text { day }\end{array}$ & $\underset{\%}{\text { Yield, }}$ & $\eta_{\text {inh }^{b}}$ & $\underset{{ }^{\circ} \mathrm{C}}{\mathrm{PMT}}$ & $\begin{array}{l}\mathrm{PMT},{ }^{\circ} \mathrm{C} \\
\text { (polyester) }\end{array}$ \\
\hline$-\left(\mathrm{CH}_{2}\right)_{2-}$ & DMF & 0.25 & 60 & 15 & 13.3 & - & - & \multirow{10}{*}{$\begin{array}{l}(102-103)^{2} \\
(88,140)^{3}\end{array}$} \\
\hline "I & DMSO & 0.25 & 60 & 50 & 66.7 & 0.10 & - & \\
\hline " & NMP & 0.50 & 202 & 1 & 87.2 & 0.14 & 80 & \\
\hline$-\left(\mathrm{CH}_{2}\right)_{4}-$ & DMF & 0.25 & 60 & 15 & 22.8 & 0.04 & 90 & \\
\hline " & $\mathrm{DMF}$ & 0.25 & 60 & 50 & 76.1 & 0.10 & 118 & \\
\hline "I & DMSO & 0.25 & 60 & 50 & 78.8 & 0.12 & - & \\
\hline " & NMP & 0.25 & 60 & 50 & 57.2 & 0.09 & - & \\
\hline$" \prime$ & NMP & 0.50 & 202 & 1 & 63.2 & 0.10 & - & \\
\hline " & DMAc & 0.25 & 60 & 50 & 75.0 & 0.10 & - & \\
\hline " & HMPA & 0.25 & 60 & 50 & 73.1 & 0.10 & - & \\
\hline $\mathrm{CH}_{3}$ & DMF & 0.25 & 60 & 50 & 15.4 & 0.10 & - & \multirow{7}{*}{$(280)^{4}$} \\
\hline$" 1$ & DMAc & 0.25 & 60 & 15 & 2.1 & - & 103 & \\
\hline " & DMSO & 0.25 & 60 & 15 & 52.6 & 0.10 & - & \\
\hline$" 1$ & DMSO & 0.25 & 60 & 50 & 94.9 & 0.15 & 152 & \\
\hline " & NMP & 0.25 & 60 & 15 & 15.6 & 0.03 & 107 & \\
\hline " & NMP & 0.50 & 202 & 1 & 71.2 & 0.13 & - & \\
\hline "I & HMPA & 0.25 & 60 & 15 & 13.8 & 0.03 & - & \\
\hline
\end{tabular}

a Catalyst, EtOLi, $5 \mathrm{~mol} \%$. b $0.05 \mathrm{~g} / 10 \mathrm{ml}$ in DMSO at $30^{\circ} \mathrm{C}$. 


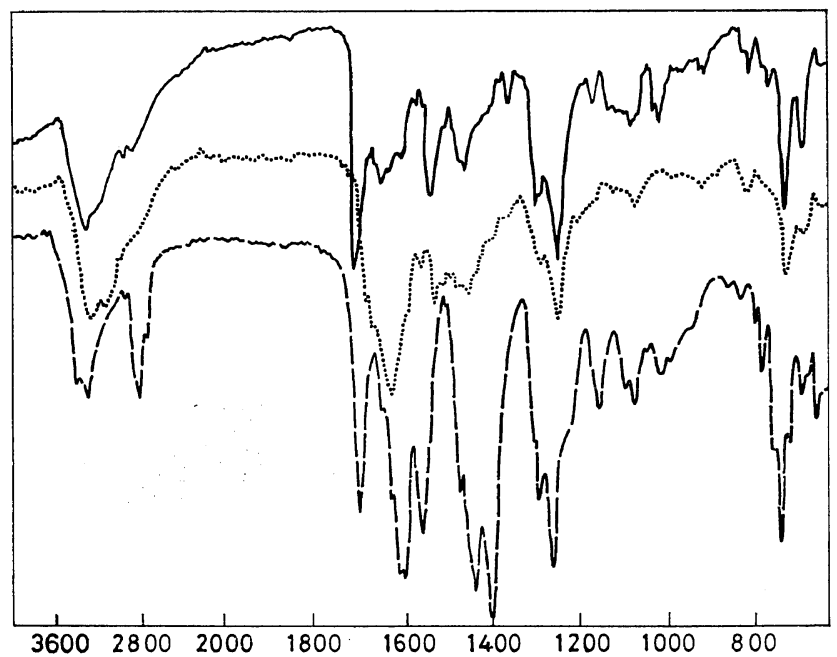

Figure 3. Infrared spectra of polythioester, polythiohydrazide, and polythiadiazole:

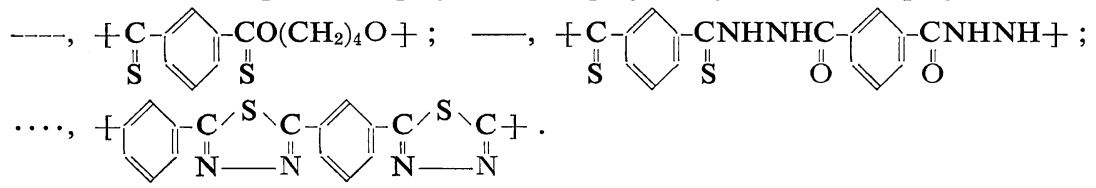

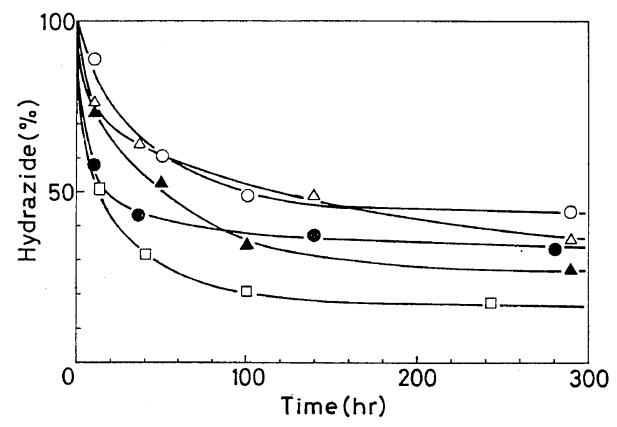

Figure 4. Rates of polycondensation of DTIP with isophthalic acid dihydrazide in various solvents at $60^{\circ} \mathrm{C}$ : O, DMF; $\triangle$, DMAc; $\square$, DMSO; $\bullet$ NMP; A, HMPA; monomer concn, $0.10 \mathrm{~mol} / l$; catalyst, ETOLi, 5 mol\%.

facilitate the polycondensation. All of the polycondensation reactions of DTIP with nucleophiles including diamines, dihydrazides or diols proceeded rapidly in DMSO. Since the polycondensation was accelerated with a base, the weak basicity of DMSO may have an accelerating effect on the polycondensation reaction. The apparent equilibrium of the polycondensation might be related to the solvation of resulting polymers,

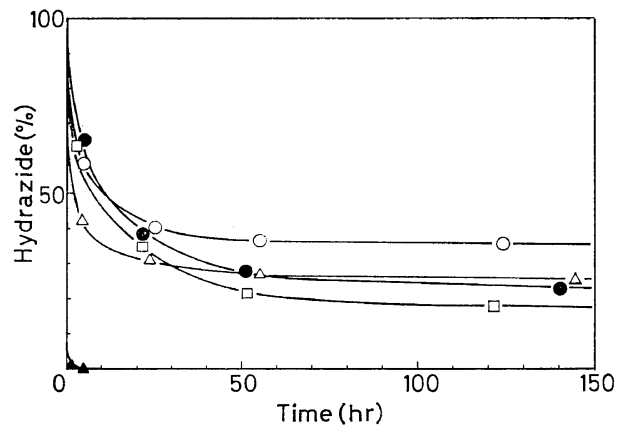

Figure 5. Rates of polycondensation of DTIP with various dihydrazides in DMSO at $60^{\circ} \mathrm{C}: \bigcirc$, $-\left(\mathrm{CH}_{2}\right)_{4^{-}} ; \triangle,-\left(\mathrm{CH}_{2}\right)_{8^{-}} ; \square,-\widehat{N}^{-} ; \bullet,-\langle$

A, Rates of polycondensation of DTIP with hexamethylenediamine under the same conditions; Monomer concn, $0.10 \mathrm{~mol} / l$; catalyst EtOLi, $5 \mathrm{~mol} \%$.

although no confirmation on this point was made.

The rates of polycondensation of DTIP with various aliphatic or aromatic dihydrazides are shown in Figure 5, which shows that the polycondensation of DTIP takes place slowly to form polythiohydrazides and that no definite differences 
Y. Kishimoto, K. SANUi, and N. Ogata

Table II. Polycondensation of DTIP with dihydrazides ${ }^{a}$

\begin{tabular}{|c|c|c|c|c|c|c|c|}
\hline $\begin{array}{c}\text { Dihydrazide } \\
\mathrm{NH}_{2} \mathrm{NHCO}^{-\mathrm{R}-\mathrm{CONHNH}}{ }_{2}\end{array}$ & Solvent & $\begin{array}{l}\text { Concn, } \\
\mathrm{mol} / l\end{array}$ & $\underset{{ }^{\circ} \mathrm{C}}{\text { Temp, }}$ & $\begin{array}{l}\text { Time, } \\
\text { day }\end{array}$ & $\begin{array}{l}\text { Yield, } \\
\%\end{array}$ & $\eta_{\mathrm{inh}}{ }^{\mathrm{b}}$ & $\begin{array}{c}\mathrm{PMT}, \\
{ }^{\circ} \mathrm{C},\end{array}$ \\
\hline$-\left(\mathrm{CH}_{2}\right)_{4}-$ & DMF & 0.25 & 153 & $10 / 24$ & 65.8 & 0.16 & 280 \\
\hline "I & NMP & 0.25 & 202 & $15 / 24$ & 86.0 & 0.23 & 274 \\
\hline " & DMSO & 0.05 & 30 & 15 & 29.9 & 0.05 & - \\
\hline " & DMSO & 0.05 & 60 & 5 & 45.8 & 0.06 & - \\
\hline " & DMSO & 0.10 & 60 & 12 & 71.8 & 0.12 & 245 \\
\hline$-\left(\mathrm{CH}_{2}\right)_{8^{-}}$ & DMF & 0.25 & 153 & $10 / 24$ & 70.2 & 0.18 & 294 \\
\hline " & NMP & 0.25 & 202 & $15 / 24$ & 93.1 & 0.37 & 276 \\
\hline " & DMSO & 0.05 & 30 & 15 & 36.0 & 0.06 & - \\
\hline " & DMSO & 0.05 & 60 & 5 & 52.4 & 0.07 & - \\
\hline " & DMSO & 0.10 & 60 & 12 & 86.0 & 0.15 & - \\
\hline & DMF & 0.10 & 60 & 20 & 59.4 & 0.15 & 305 \\
\hline " & DMF & 0.25 & 153 & $10 / 24$ & 83.2 & 0.14 & 315 \\
\hline$"$ & DMAc & 0.10 & 60 & 20 & 64.3 & 0.16 & 303 \\
\hline " & NMP & 0.10 & 60 & 20 & 65.2 & 0.15 & 305 \\
\hline " & NMP & 0.25 & 202 & $15 / 24$ & 92.5 & 0.28 & 309 \\
\hline " & DMSO & 0.05 & 30 & 15 & 63.1 & 0.04 & 267 \\
\hline " & DMSO & 0.05 & 60 & 5 & 54.5 & 0.07 & - \\
\hline " & DMSO & 0.10 & 60 & 12 & 70.4 & 0.13 & - \\
\hline " & DMSO & 0.10 & 60 & 20 & 82.7 & 0.14 & 324 \\
\hline " & HMPA & 0.10 & 60 & 20 & 72.8 & 0.16 & 308 \\
\hline & DMF & 0.25 & 153 & $10 / 24$ & 84.2 & 0.13 & 309 \\
\hline$"$ & NMP & 0.25 & 202 & $15 / 24$ & 87.3 & 0.29 & 305 \\
\hline$" 1$ & DMSO & 0.05 & 30 & 15 & 47.0 & 0.05 & - \\
\hline " & DMSO & 0.05 & 60 & 5 & 49.1 & 0.07 & - \\
\hline " & DMSO & 0.10 & 60 & 12 & 74.8 & 0.13 & - \\
\hline
\end{tabular}

a Catalyst, EtOLi, $5 \mathrm{~mol} \%$. ${ }^{\mathrm{b}} 0.05 \mathrm{~g} / 10 \mathrm{ml}$ in DMSO at $30^{\circ} \mathrm{C}$.

in reactivity between aliphatic or aromatic dihydrazides are found. From these experiments, the order of the reaction rate of DTIP with nucleophiles can be shown to be as follows: diamine $\gg$ dihydrazide $>$ diol. The results of the polycondensation of DTIP with various dihydrazides under various reaction conditions are summarized in Table II, which indicates that the reaction takes place easily with rising temperatures to form polythiohydrazide with a relatively high solution viscosity.

All of the polythiohydrazides were soluble in DMSO and no cross-linking reaction occured even when the polymer solution is heated to an elevated temperature. When the polythiohydrazide was heated to temperatures above $230^{\circ} \mathrm{C}$, an intramolecular dehydration reaction occured with a ring closure to form polythiadiazole as pre- viously reported by Frazer $^{5}$ as shown in Figure 3 , where it is seen that the absorptions due to carbonyl groups disappear on heating. No detailed study on the thermal stability of resulting polythiadiazole was made since Frazer had reported in detail on this problem.

\section{CONCLUSION}

The derivative of carboxylic acid that is most reactive towards nucleophiles such as amine or alcohol is, as is well known, carboxylic acid chloride and polycondensation reactions of dicarboxylic acid chlorides with diamines generally go to completion within $10 \mathrm{~min} .{ }^{6}$ Although thiocarboxylic acid $O$-alkyl ester can react with nucleophiles to form corresponding products in a good yield, its reactivity toward nucleophiles 
is lower than that of the acid chloride since the reaction with amine requires $5 \mathrm{hr}$ for the completion at room temperature and it takes more than several months in the reaction with hydrazide or alcohol.

However, the reactivity of the thioester is much higher than that of aliphatic esters, presumably due to the polar effect of the thiocarbonyl group.

The chelating effect of metal catalysts on this polycondensation is an interesting theme to study with reference to the polarity contribution of the thiocarbonyl group and the study of this problem is in progress.

\section{REFERENCES}

1. N. Ogata, Y. Kishimoto, and K. Sanui, Polymer J., 2, 789 (1971).

2. F. W. Knobloch and W. H. Rauscher, J. Polym. Sci., 54, 651 (1961).

3. R. Hill, "Fibre from Synthetic Polymers" Elsevier, London, 1953.

4. W. M. Eareckson, J. Polym. Sci., 40, 399 (1959).

5. A. H. Frazer and W. P. Fitzgerald, J. Polym. Sci., Part C, 24, 25 (1968).

6. P. W. Morgan, "Condensation Polymers by Interfacial and Solution Methods", Interscience New York, N. Y., 1965. 